\title{
Host Fish Identification and Early Life Thermal Requirements for the Federal Endangered Winged Mapleleaf Mussel
}

The winged mapleleaf mussel (Quadrula fragosa, WML) is a Federal endangered species historically inhabiting at least 34 river systems in 12 Midwestern states. Only four populations are currently known to exist, including one confirmed reproducing population in the St. Croix National Scenic Riverway (NSR) bordering Minnesota and Wisconsin. Recovery efforts are limited by a lack of life history information, particularly which species of fish serve as host to the mussel's parasitic larvae (glochidia).

Since 1997, biologists at the University of Minnesota have tried to identify host fish for the WML. Department of the Interior colleagues working in western Wisconsin at the U.S. Fish and Wildlife Service's La Crosse Fishery Resources Office and Genoa National Fish Hatchery, the National Park Service's St. Croix NSR, and the U.S. Geological Survey's Upper Midwest Environmental Sciences Center (UMESC) in La Crosse joined the team in 2001 to expand and accelerate the laboratory host fish identification program by making use of the well-equipped aquatic research facilities at UMESC. We report on several WML early life history investigations conducted at UMESC since autumn 2003 to identify suitable host fish species and evaluate thermal requisites for the development of glochidia into free-living juvenile mussels.

A series of WML host-fish identification trials were conducted with as many as four species of the catfish family (Ictaluridae) including blue catfish (Ictalurus furcatus; BCF), channel catfish (I. punctatus; CCF), flathead catfish (Pylodictus olivarus; FHC), and slender madtom (Noturus exilis; SMT). On four dates in autumn 2003, fish were infested with viable glochidia from several winged mapleleaf mussels from the St. Croix River.

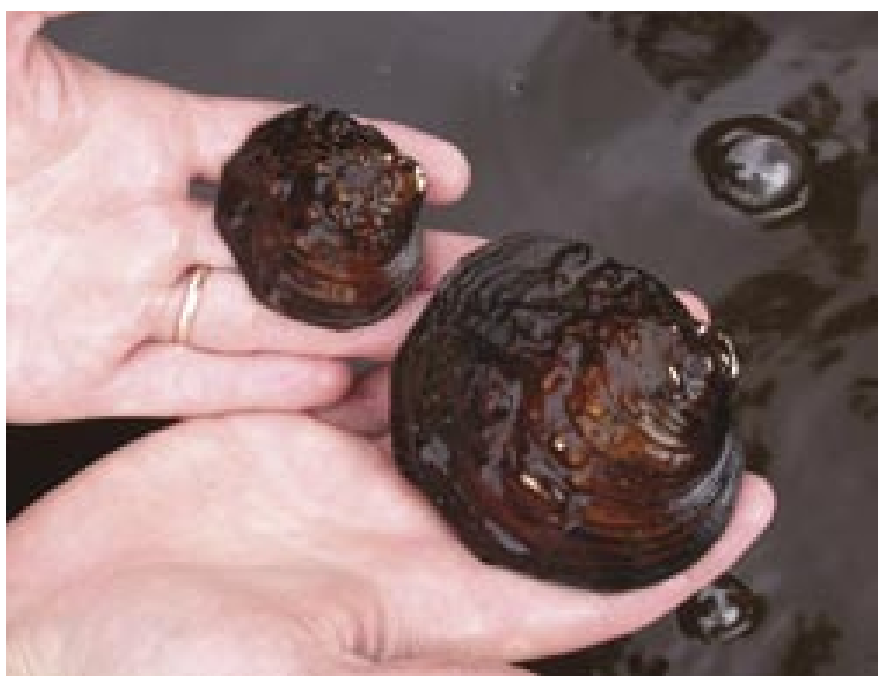

The only known reproducing population of winged mapleleaf mussels (Quadrula fragosa) inhabits the St. Croix National Scenic Riverway.
One group of BCF and one group of CCF (5 fish/group) were infested each day by placing them in $2 \mathrm{~L}$ of vigorously aerated well water innoculated with about $150 \mathrm{ml}$ of a glochidial slurry. Groups of SMT and FHC (2 fish/group) were infested by pipetting glochidia $(5-15 \mathrm{~mL})$ directly onto the gills.

Infested fish were then assigned to one of three water temperatures (Test I, II, or III):

Test $I$ - fish were maintained $49 \mathrm{~d}$ in aquaria that received a continuous flow of $19.6^{\circ} \mathrm{C}$ water;

Test II - one group of CCF was maintained $45 \mathrm{~d}$ at $12.5^{\circ} \mathrm{C}$, then acclimated and placed in aquaria that received a continuous flow of $19.5^{\circ} \mathrm{C}$ water;

Test III - one group of CCF was maintained $45 \mathrm{~d}$ at $12.5^{\circ} \mathrm{C}$, then held in a thermal regime similar to the St. Croix River from November 2003 to June 2004.

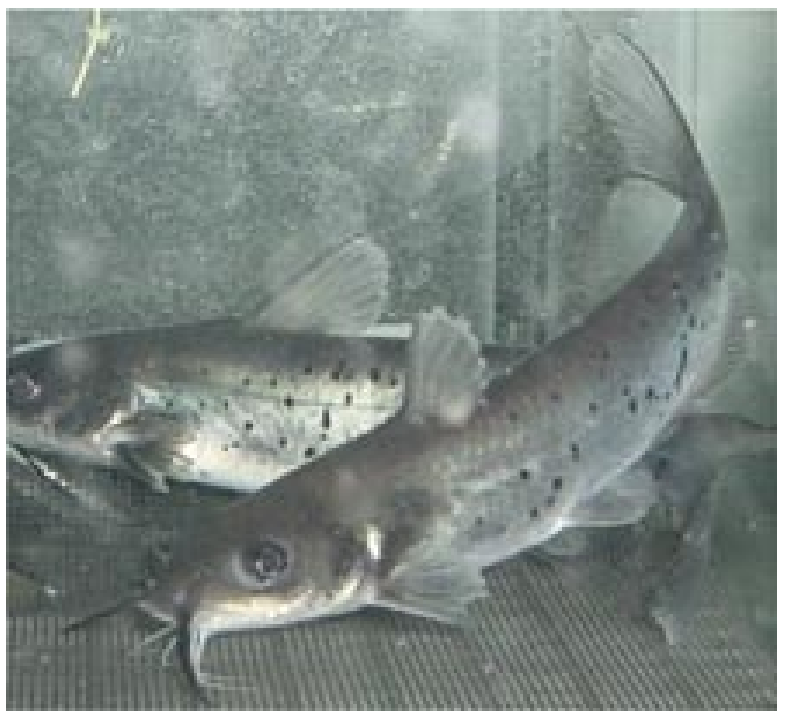

Fish were kept in laboratory aquaria during all tests to monitor the daily excystment of juvenile winged mapleleaf mussels (Quadrula fragosa).

Water siphoned from the aquaria each day was filtered through a series of sieves $(53,153$, and $202 \mu \mathrm{m}$ mesh). Materials retained on sieves were illuminated with cross-polarized light and examined with a dissection microscope to enumerate mussels. Juvenile mussels were considered viable if either external (foot, valve) or internal (ciliary) movement was observed. The consecutive 5-d period during each test when the maximum number of viable juveniles was recovered from an aquarium was considered the period of peak juvenile excystment.

About 11,600 viable juveniles were recovered within 7 weeks from groups of $\mathrm{BCF}$ and $\mathrm{CCF}$ held at a constant temperature of $19.6^{\circ} \mathrm{C}$ (Test I). Production by BCF accounted for $95 \%$ of this total. Peak recovery from CCF and BCF occurred 4-5 weeks 
and 5-6 weeks after infestation, respectively. The rate at which glochidia successfully transformed into viable juveniles was $50 \%$ on BCF, but only $7 \%$ on CCF. Juveniles were not recovered from FHC or SMT. These results suggested that BCF is a suitable host whereas CCF was a marginal host.

In Test II, about 9,300 viable juveniles were recovered from the CCF after 11 weeks of development in temperatures that ranged 11.8$19.7^{\circ} \mathrm{C}$ (Test II). When compared to the results from Test I, this was a 64-fold increase in the mean number of juveniles produced per CCF. A combination of factors may explain this difference. First, the mean weight of CCF used in Test II (156 g) was much greater than that of CCF (18 g) and BCF (60 g) used in Test I. This likely provided more gill surface area on which glochidia could encyst. Second, more glochidia were used to infest fish for Test II than for Test I. Overall, these results suggest CCF is also a suitable host. This finding is useful to managers wanting to develop propagation strategies to recover WML populations throughout its historical range, including the St. Croix and other northern locations where CCF are often present year-round and BCF are usually absent or rare.

After fish were maintained in warm water $\left(19.5^{\circ} \mathrm{C}\right)$ such as that used throughout Test I, peak recovery of juveniles from CCF during Test II occurred about one week earlier than it did during Test I. This finding suggested the physiological mechanisms responsible for transformation of glochidia into juveniles on CCF proceeded more slowly at colder temperatures.

A species-specific temperature threshold for early life development of poikilotherms can be empirically derived from repeated embryological observations made over an environmentally relevant temperature range. Given a complete

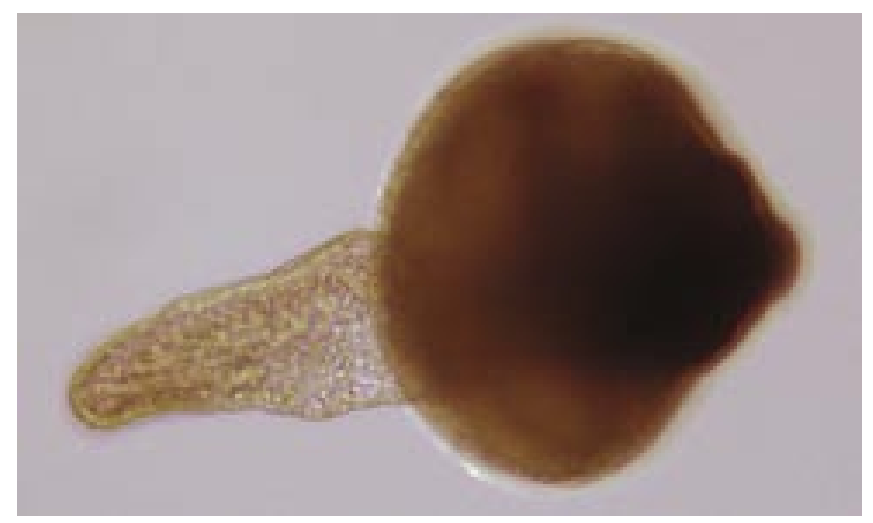

A juvenile winged mapleleaf mussel (Quadrula fragosa; foot extended) recovered after 266 days of development in a simulated St. Croix River thermal regime and $<24$ hours after excystment from its channel catfish host (/ctalurus punctatus).

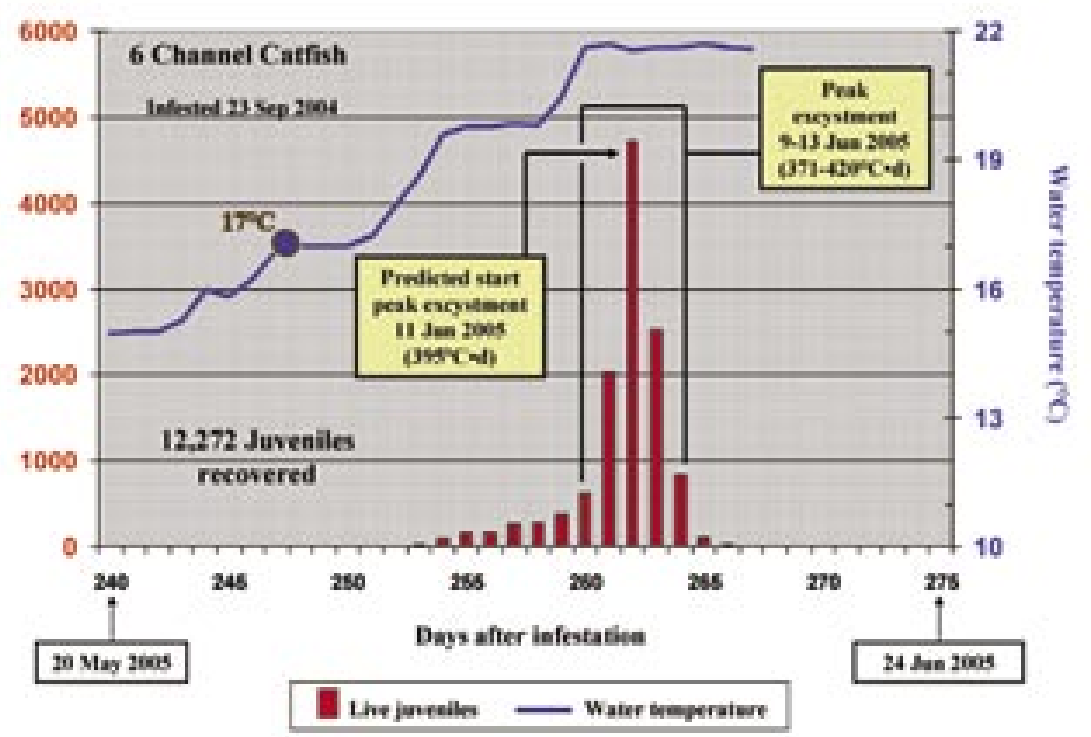

Recovery of juvenile winged mapleleaf mussels (Quadrula fragosa) from channel catfish (Ictalurus punctatus) reared in a simulated St. Croix River thermal regime, September 2004June 2005.

record of the mean daily water temperature and the number of juveniles recovered daily from each aquarium during Tests I and II, we estimated the temperature threshold for development of $\mathrm{CCF}$-encysted glochidia into juveniles to be $9.24^{\circ} \mathrm{C}$.

The validity of this estimate was evaluated during Test III. Peak excystment began 21 June when WML attained an estimated $453^{\circ} \mathrm{C} \cdot \mathrm{d}$ of development. Based on the results of Tests I and II, peak excystment during Test III was predicted to start 14 June when $395^{\circ} \mathrm{C} \bullet \mathrm{d}$ of development was attained. Thus, we successfully predicted the dates of peak excystment to within 1 week during a 37-week developmental period, representing an error rate of $<3 \%$. As the predicted dates of peak excystment came and passed, few juveniles were recovered at cold test temperatures $\left(<15^{\circ} \mathrm{C}\right)$, but warmer temperatures $\left(17-20^{\circ} \mathrm{C}\right)$ later appeared to act as a thermal cue and initiate excystment. This test was repeated the following year (September 2004 - June 2005) when peak excystment was predicted to begin 11 June but actually began 9 June, representing an error rate of $<1 \%$. This test also provided supporting evidence for the $17-20^{\circ} \mathrm{C}$ thermal cue that we speculate is needed to trigger excystment.

These results confirm the validity of our approach to quantify the period of thermal development necessary for transformation of WML glochidia into juveniles on CCF and present a model to quantify temperature dependent development for other mussel species. This information will aid recovery teams in establishing successful propagation programs for mussels with historically wide-ranging distributions and multiple hosts (such as the WML) and can be used to improve juvenile mussel production for other listed species with existing propagation programs.

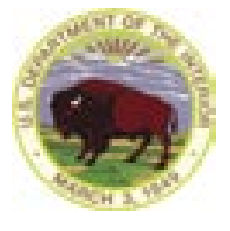

